\title{
Intuitionistic fuzzy prime ideals of near-subtraction semigroups
}

\author{
P. Murugadas ${ }^{1 *}$
}

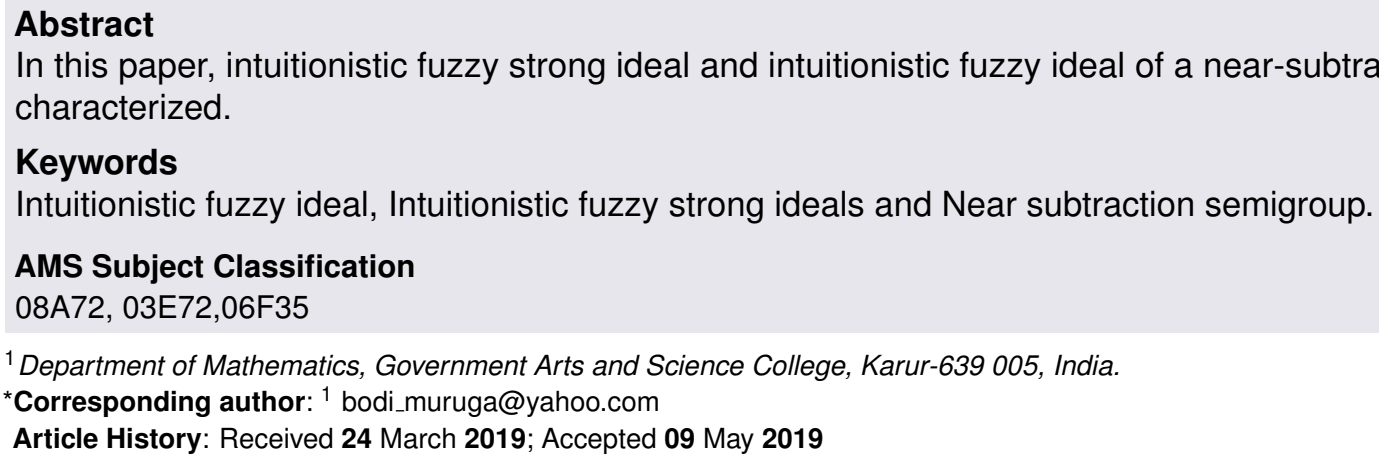

\section{Contents}

1 Introduction ............................. 139

2 Preliminaries ........................... 140

3 Intuitionistic Fuzzy Strong ideal of Near-ring ..... 140

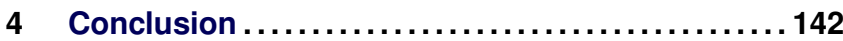

References ............................... 142

\section{Introduction}

Zadeh [17] introduced the concept of fuzzy sets in 1965. The notion of fuzzy subgroup was made by Rosenfeld [13] (1971). Liu [9], (1982), introduced the notion of fuzzy ideal of a ring. The notions of fuzzy sub near-ring, fuzzy ideal and fuzzy $N$-subgroup of a near-ring was introduced by Salah Abou-Zaid [1] (1991).

The concept of bi-ideals was applied to near-rings in [16]. Manikantan [12] introduced the notion of fuzzy bi-ideals of near-rings and discussed some of its properties. B.M Schein [15] considered a system $(\phi ; \circ, \backslash)$ as a set of functions closed under the composition "० " of function and the subtraction " $\backslash$ as in set theory. He showed that each subtraction semigroup is isomorphic to a difference semigroup of a function which is invertible. The problem proposed by B.M Schein on the structure of multiplication in a subtraction semigroup was discussed by B.Zelinka [18]. Jun et.al, [8] initiated the work on ideals in subtraction algebra and characterized the ideals in it. Prime and fuzzy prime ideals of subtraction algebra and near-subtraction semigroup were introduced by Dheena et.al, $[5,6]$.

The concept of Intuitionistic Fuzzy Set(IFS) was introduced by Atanassov [2] as a generalization of the notion of fuzzy set. Biswas [3] applied the concept of IFSs to the groups and studied nItuitionistic Fuzzy (IF)subgroups of a group. The notion of an IF ideal of a near ring is given by Jun, Kim and Yon [7]. Also Cho and Jun [4] introduced the notion of IF $R$-subgroups in a near-rings and analyzed some properties. Kazanci, Yamak and Yilmaz [10] introduced intuitionistic $Q$-fuzzy $R$-subgroups of near-rings. Kim [11] introduced intuitionistic $Q$-fuzzy semiprime ideals of semigroups.

A nonempty set $X$ with a binary operation ' ${ }^{\prime}$ satisfying the following $(i) \cdot x-(y-x)=x,(i i) \cdot x-(x-y)=y-(y-x)$ and (iii). $(x-y)-z=(x-z)-y$ for all $x, y, z \in X$ is called a subtraction algebra and the following are true in any subtraction algebra. $1 . x-0=x$ and $0-x=0.2 \cdot(x-y)-x=0$. 3. $(x-y)-y=x-y \cdot 4 \cdot x-(x-y) \leq y \cdot 5 \cdot(x-y)-(y-x)=$ $x-y .6 \cdot x-(x-(x-y))=x-y .7 .(x-y)-(z-y) \leq x-z$. 8. $x \leq y$ iff $x=y-w$ for some $w \in X .9 . x \leq y$ implies $x-z \leq$ $y-z$ and $z-y \leq z-x$ for all $z \in X .10 . x, y \leq z$ implies $x-y=x \wedge(z-y)$. In addition with ${ }^{\prime}{ }^{\prime}$ operation, if $X$ satisfies the following with'.' operation 1. $(X ;-)$ is a subtraction algebra. 2. $(X ;$.$) is a semigroup and 3.x (y-z)=x y-x z$ and $(x-y) z=x z-y z$ for all $x, y, z \in X$ is called a subtraction semigroup. It is also clear that $0 x=0$ and $x 0=0$ for all $x \in X$. For any two nonempty subset $A, B \in X, A B=\{a b \mid a \in A, b \in B\}$. A subtraction semigroup is called a near subtraction semigroup if $x(y-z)=x y-x z$ or $(x-y) z=x z-y z$ is true. In a near-subtraction semigroup $X$ if $x 0=0$ for all $x \in X$, then $X$ is said to zero- symmetric. A nonempty subset $I$ of a subtraction 
algebra $X$ is said to be a subalgebra of $X$, if $x-y \in I$ for all $x, y \in I$. A nonempty subset $I$ of a near-subtraction semigroup is called $I 1$. a left ideal of $X$ if $I$ is a subalgebra of $(X,-)$ and $x i-x(y-i) \in I$ for all $x, y \in X$ and $i \in I$. I2 a right ideal if $I$ is a subalgebra of $(X,-)$ and $I X \subseteq I$. and $I 3 I$ is an ideal if $I$ is both left and right ideal. If $N$ is zero-symmetric then $x i-x(y-i) \in I$ for all $x, y \in X$ and $i \in I$. if and only if $X I \subseteq I$.

In this paper, we introduce the notion of intuitionistic $Q$-fuzzy strong bi-ideal of a near-ring and obtain the characterization of a strong bi-ideal in terms of a intuitionistic $Q$-fuzzy strong bi-ideal of a near-ring.

\section{Preliminaries}

In what follows, let $N$ denote a near-subtraction algebra.

Definition 2.1. A strong left ideal $P$ of $N$ is called a prime left ideal if $I J \subseteq P$ implies $I \subseteq P$ or $J \subseteq P$ for all left ideals $I, J$.

Definition 2.2. A strong left ideal $P$ of $N$ is called a weakly prime left ideal if $I J \subseteq P$ implies $I=P$ or $J=P$ for all left ideals $I, J$.

Definition 2.3. Let $X$ be an non-empty fixed set. An intuitionistic fuzzy set (briefly, IFS) $A$ is object having the form $A=\left\{\left\langle x, \mu_{A}(x), v_{A}(x)\right\rangle: x \in X\right\}$

where the functions $\mu_{A}: N \rightarrow[0,1]$ and $v_{A}: N \rightarrow[0,1]$ denote the degree of membership (namely $\mu_{A}(x)$ ) and the degree of non-membership (namely $v_{A}(x)$ ) of each element $x \in N$ to the set $A$, respectively, and $\mu_{A}(x)+v_{A} \leq 1$ for all $x \in N$. For the sake of simplicity, we use the symbol $A=\left\langle\mu_{A}, \lambda_{A}\right\rangle$ for the IFS $A=\left\{\left\langle x, \mu_{A}(x), v_{A}(x)\right\rangle: x \in N\right\}$.

Definition 2.4. An IFS $A$ in $N$ is called an IF ideal of $N$ if the following conditions are satisfied: (IFI1) $\mu_{A}(x-y) \geq$ $\min \left\{\mu_{A}(x), \mu_{(y)}\right\}$ and $v_{A}(x-y) \leq \max \left\{v_{A}(x), v_{A}(y)\right\}$, for all $x, y \in N$,

$\left(\right.$ IFI2) $\mu_{A}(a x-a(b-x)) \geq \mu_{A}(x)$ and $v_{A}(a x-a(b-x)) \leq$ $v_{A}(x)$ for all $a, b, x \in N$ and

$(I F I 3) \mu_{A}(x y) \geq \mu_{A}(x)$ and $v_{A}(x y) \leq v_{A}(x)$ for all $x, y \in N$,.

If (IFI1) and (IFI2) are satisfied then $A$ is an IF left ideal of $N$ and if (IFII) and (IFI3) are satisfied then $A$ is called an IF right ideal of $N$

\section{Intuitionistic Fuzzy Strong ideal of Near-ring}

Definition 3.1. A non-empty subset I of a near-subtraction semigroup $N$ is called a strong ideal if

(IFSI1). $x-y \in I$ for all $x \in I$ and for all $y \in N$.

(IFSI2). $x i-x(y-i) \in I$ for all $x, y \in N$ and for all $i \in I$.

(IFSI3). IX $\subseteq I$.

If (IFSI1) and (IFSI2) are true, then I is a strong left ideal of $N$, and (IFSI1)and (IFSI2) are true then I is a strong right ideal of $N$.
Remark 3.2. Every strong ideal of $N$ is an ideal of $N$, but the converse need not be true.

Remark 3.3. A prime left ideal is always a weakly prime left ideal and the converse is not true. It is illustrated in the following example.

Example 3.4. Let $N=\{0, x, y, z\}$ be a near-subtraction semigroupg with two binary operations ' ${ }^{\prime}$ and '! is defined as follows.

\begin{tabular}{|c|c|c|c|c|}
\hline- & 0 & $x$ & $y$ & $z$ \\
\hline 0 & 0 & 0 & 0 & 0 \\
\hline$x$ & $x$ & 0 & $z$ & $y$ \\
\hline$y$ & $y$ & 0 & 0 & $y$ \\
\hline$z$ & $z$ & 0 & $z$ & 0 \\
\hline
\end{tabular}

\begin{tabular}{|c|c|c|c|c|}
\hline$\cdot$ & 0 & $x$ & $y$ & $z$ \\
\hline 0 & 0 & 0 & 0 & 0 \\
\hline$x$ & 0 & $x$ & $y$ & $z$ \\
\hline$y$ & 0 & 0 & 0 & 0 \\
\hline$z$ & 0 & $x$ & $y$ & $z$ \\
\hline
\end{tabular}

Here the strong left ideal $\{0, z\}$ is weakly prime left ideal but it is not a prime left ideal, because $\{0, y\} \nsubseteq\{0, z\}$ is a strong left ideal such that $\{0, y\} .\{0, y\} \subseteq\{0, z\}$.

Theorem 3.5. Let $A$ be an IFS of a near-subtraction semigroup $N$. Then $A$ is an IF ideal iff the level subset $A_{\left(t, t^{\prime}\right)}$ is an ideal of $N$ for all $\left(t, t^{\prime}\right) \in[0,1]^{2}$ with $t+t^{\prime} \leq 1$ whenever nonempty.

Proof. Let $A$ be an IF ideal of a near-subtraction semigroup $N$. Let $\left(t, t^{\prime}\right) \in[0,1]^{2}$ with $t+t^{\prime} \leq 1$ and $x, y \in A_{\left(t, t^{\prime}\right)}$. Then $\mu_{A}(x-y) \geq \min \left\{\mu_{A}(x), \mu_{A}(y)\right\} \geq t$ and

$v_{A}(x-y) \leq \max \left\{v_{A}(x), v_{A}(y)\right\} \leq t^{\prime}$. Thus $x-y \in \in A_{\left(t, t^{\prime}\right)}$ for all $x, y \in A_{\left(t, t^{\prime}\right)}$. If $x \mathbf{1} n A_{\left(t, t^{\prime}\right)}$, then $\mu_{A}(z x-z(y-x)) \geq \mu_{A}(x) \geq t$ and $v_{A}(z x-z(y-x)) \leq v_{A}(x) \leq t^{\prime}$. Further for $x, y \in A_{\left(t, t^{\prime}\right)}$, $\mu_{A}(x y) \geq \mu_{A}(x) \geq t$ and $v_{A}(x y) \leq v_{A}(x) \leq t^{\prime}$, for all $x \in A_{\left(t, t^{\prime}\right)}$ and $y, z \in A_{\left(t, t^{\prime}\right)}$. Thus $A_{\left(t, t^{\prime}\right)}$ is an ideal for all $\left(t, t^{\prime}\right) \in[0,1]^{2}$ with $t+t^{\prime} \leq 1$ whenever nonempty. Conversely, let $x, y z \in N$. For $t=\min \left\{\mu_{A}(x), \mu_{A}(y)\right\}$ and $t^{\prime}=\max \left\{v_{A}(x), v_{A}(y)\right\}, x, y \in$ $A_{\left.t, t^{\prime}\right)}$. As $x-y \in A_{t, t^{\prime}}, \mu_{A}(x-y) \geq \min \left\{\mu_{A}(x), \mu_{A}(y)\right\}=t$ and $v_{A}(x-y) \leq \max \left\{v_{A}(x), v_{A}(y)\right\}=t^{\prime}$. Let $\left(t, t^{\prime}\right)=A(x)$, then $x \in A_{\left(t, t^{\prime}\right)}$ and $z x-z(y-x), x y \in A(x)=\left(\mu_{A}(x), v_{A}(x)\right)$ for all $y, z \in N$. Therefore $\mu_{A}(z x-z(y-x)) \geq \mu_{A}(x)=t, v_{A}(z x-$ $z(y-x)) \leq v_{A}(x)=t^{\prime}$ and $\mu_{A}(x y) \geq \mu_{A}(x)=t$, $v_{A}(x y) \leq v_{A}(x)=t^{\prime}$. Thus $A$ is an IF ideal of $N$.

Theorem 3.6. Let $A$ be an IFS of a near-subtraction semigroup $N$. Then $A$ is an IF strong ideal iff the level subset $A_{\left(t, t^{\prime}\right)}$ is an ideal of $N$ for all $\left(t, t^{\prime}\right) \in[0,1]^{2}$ with $t+t^{\prime} \leq 1$ whenever nonempty.

Proof. Let $A$ be an IF strong ideal of a near-subtraction semigroup $N$. Let $\left(t, t^{\prime}\right) \in[0,1]^{2}$ with $t+t^{\prime} \leq 1$. If $x \in A_{\left(t, t^{\prime}\right)}$, then $\mu_{A}(x-y) \geq \mu_{A}(x) \geq t, v_{A}(x-y) \leq v_{A}(x) \leq t^{\prime}, \quad \mu_{A}(z x-$ $z(y-x) \geq \mu_{A}(x) \geq t, v_{A}\left(z x-z(y-x) \leq v_{A}(x) \leq t^{\prime}, \quad \mu_{A}(x y) \geq\right.$ $\mu_{A}(x) \geq t v_{A}(x y) \leq v_{A}(x) \leq t^{\prime}$. Thus $x-y, z x-z(y-x), x y \in$ $A_{\left(t, t^{\prime}\right)}$ for all $x \in A_{\left(t, t^{\prime}\right)}$ and for all $y, z \in N$. Therefore $A_{\left(t, t^{\prime}\right)}$ is a strong ideal of $N$. 
Conversely, let $x, y, z \in N$. Let $\left(t, t^{\prime}\right)=\left(\mu_{A}(x), v_{A}(x)\right)$. Now $x \in A_{\left(t, t^{\prime}\right)}$. Therefore $x-y, z x-z(y-x), x y \in A_{\left(t, t^{\prime}\right)}$ for all $y, z \in$ $N$. Thus $\mu_{A}(x-y) \geq \mu_{A}(x)=t, \mu_{A}\left(z x-z(y-x) \geq \mu_{A}(x)=t\right.$ and $\mu_{A}(x y) \geq \mu_{A}(x)=t, v_{A}(x-y) \leq v_{A}(x)=t^{\prime}, v_{A}(z x-z(y-$ $x) \leq v_{A}(x)=t^{\prime}$ and $v_{A}(x y) \leq v_{A}(x)=t^{\prime}$. Therefore $A$ is a IF strong ideal of $N$.

\section{Corollary 3.7. Every IF strong ideal is IF ideal.}

However the converse of the above corollary is not true in general as shown in the following example.

Example 3.8. Let $N=\{0, x, y, z\}$ be a near-subtraction semigroupg with two binary operations ' - ' and '! is defined as follows.

\begin{tabular}{|c|c|c|c|c|}
\hline- & 0 & $x$ & $y$ & $z$ \\
\hline 0 & 0 & 0 & 0 & 0 \\
\hline$x$ & $x$ & 0 & $z$ & $y$ \\
\hline$y$ & $y$ & 0 & 0 & $y$ \\
\hline$z$ & $z$ & 0 & $z$ & 0 \\
\hline
\end{tabular}

\begin{tabular}{|c|c|c|c|c|}
\hline$\cdot$ & 0 & $x$ & $y$ & $z$ \\
\hline 0 & 0 & 0 & 0 & 0 \\
\hline$x$ & 0 & $x$ & $x$ & $x$ \\
\hline$y$ & $x$ & 0 & $z$ & $y$ \\
\hline$z$ & 0 & $x$ & $y$ & $z$ \\
\hline
\end{tabular}

$$
\begin{aligned}
& \mu_{A}(a)= \begin{cases}0.8 & \text { if } a \in\{0, x\} \\
0.3 & \text { otherwise }\end{cases} \\
& v_{A}(a)= \begin{cases}0.1 & \text { ifa } \in\{0, x\} \\
0.6 & \text { otherwise }\end{cases}
\end{aligned}
$$

Clearly $A=\left(\mu_{A}(x), v_{A}(x)\right)$ is a IF right ideal of $N$, but not a $I F$ right strong ideal of $N$, since $\mu_{A}(x-y=z)=0.3<0.9=$ $\mu_{A}(x)$.

Definition 3.9. The product of two IFSs $A=\left(\mu_{A}, v_{A}\right)$ and $B=\left(\mu_{B} . v_{B}\right)$ of $N$ is defined as

$$
(A . B)(x)=\left\{\begin{array}{l}
\left(\sup _{x=y z} \min \left\{\mu_{A}(y), \mu_{B}(z)\right\},\right. \\
\left.\inf _{x=y z} \max \left\{v_{A}(y), v_{B}(z)\right\}\right) \quad \text { if } x=y z \\
(0,1) \text { otherwise }
\end{array}\right.
$$

Definition 3.10. An IF strong ideal A of a near-subtraction semigroup $N$ is called IF prime if $C . D \subseteq A$ implies $C \subseteq A$ or $D \subseteq A$ for all IF strong ideals $C, D$ of $N$.

Definition 3.11. An IF strong ideal A of a near-subtraction semigroup $N$ is called IF weakly prime if $C . D \subseteq A$ implies $C=A$ or $D=A$ for all IF strong ideals $C, D$ which contains $N$.

Any IF prime left ideals is an if weakly prime ideal. However the converse is not true, it is shown in the following example.

Example 3.12. Let us consider the $N$ in Example 3.4 and define IFS $A=\left(\mu_{A}, v_{A}\right), B=\left(\mu_{B}, v_{B}\right)$ as

$\mu_{A}(x)=\left\{\begin{array}{l}1 \quad \text { if } x \in\{0, z\} \\ 0.2 \text { otherwise }\end{array} \quad v_{A}(x)=\left\{\begin{array}{lr}0 & \text { if } x \in\{0, z\} \\ 0.7 & \text { otherwise }\end{array}\right.\right.$

$$
\mu_{B}(x)=\left\{\begin{array}{l}
0.8 \text { if } x \in\{0, y\} \\
0 \text { otherwise }
\end{array}\right.
$$

$v_{B}(x)=\left\{\begin{array}{l}0.1 \quad \text { if } x \in\{0, y\} \\ 1 \quad \text { otherwise }\end{array} \quad \mu_{(B . B)}(x)= \begin{cases}1 & \text { if } x \in\{0\} \\ 0 & \text { otherwise }\end{cases}\right.$

$$
v_{B . B}(x)= \begin{cases}0 & \text { if } x \in\{0\} \\ 1 & \text { otherwise }\end{cases}
$$

Here the IF strong left ideal $A$ is IF weakly prime left ideal but not IF prime left ideal, because $B . B \subseteq A$ but $B \nsubseteq A$.

Lemma 3.13. Let $A$ be a non constant IF weakly prime left ideal of $N$, then $\operatorname{Im} A=\left\{(1,0),\left(t, t^{\prime}\right)\right\}$ with $t+t^{\prime} \leq 1$, where $(1,0)>\left(t, t^{\prime}\right) \geq(0,1)$.

Proof. If $\operatorname{Im} A=\left\{\left(t_{1}, t_{1}^{\prime}\right),\left(t_{2}, t_{2}^{\prime}\right),\left(t_{3}, t_{3}^{\prime}\right)\right\}$ for $(1,0)>\left(t_{1}, t_{1}^{\prime}\right)>$ $\left(t_{2}, t_{2}^{\prime}\right)>\left(t_{3}, t_{3}^{\prime}\right) \geq(0,1)$, then there exist $a, b, c \in N$ such that $\left(\mu_{A}(a), v_{A}(a)\right)=\left(t_{1}, t_{1}^{\prime}\right),\left(\mu_{A}(b), v_{A}(b)\right)=\left(t_{2}, t_{2}^{\prime}\right),\left(\mu_{A}(c), v_{A}(c)\right)$ $=\left(t_{3}, t_{3}^{\prime}\right)$. Choose $\left(s_{1}, s_{1}^{\prime}\right),\left(s_{2}, s_{2}^{\prime}\right)$ such that $(1,0)>\left(s_{1}, s_{1}^{\prime}\right)>$ $\left(t_{1}, t_{1}^{\prime}\right)>\left(s_{2}, s_{2}^{\prime}\right)>\left(t_{2}, t_{2}^{\prime}\right)$. Define $C(x)=\left(\mu_{C}(x), v_{C}(x)\right)$ and $D(x)=\left(\mu_{D}(x), v_{D}(x)\right)$ as

$$
\begin{aligned}
& C(x)= \begin{cases}\left(s_{1}, s_{1}^{\prime}\right) & \text { if } x \in A_{\left(t_{1}, t_{1}^{\prime}\right)} \\
\left(t_{2}, t_{2}^{\prime}\right) & \text { otherwise }\end{cases} \\
& D(x)= \begin{cases}\left(t_{1}, t_{1}^{\prime}\right) & \text { if } x \in A_{\left(t_{1}, t_{1}^{\prime}\right)} \\
\left(s_{2}, s_{2}^{\prime}\right) & \text { if } x \in A_{\left(t_{2}, t_{2}^{\prime}\right)}-A_{\left(t_{1}, t_{1}^{\prime}\right)} \\
\left(t_{3}, t_{3}^{\prime}\right) & \text { otherwise }\end{cases}
\end{aligned}
$$

Clearly $A \subseteq C, A \subseteq D$.

$$
(D . C)(x)=\left\{\begin{array}{lll}
\left(t_{1}, t_{1}^{\prime}\right) & \text { if } x=y z & y, z \in A_{\left(t_{1}, t_{1}^{\prime}\right)} \\
\left(s_{2}, s_{2}^{\prime}\right) & \text { if } x=y z, & y \in A_{\left(t_{2}, t_{2}\right)}-A_{\left(t_{1}, t_{1}^{\prime}\right)}, z \in A_{\left(t_{1}, t_{1}^{\prime}\right)} \\
\left(t_{2}, t_{2}^{\prime}\right) & \text { if } x=y z, & y, z \in A_{\left(t_{2}, t_{2}^{\prime}\right)}-A_{\left(t_{1}, t_{1}^{\prime}\right)} \\
\left(t_{3}, t_{3}^{\prime}\right) & \text { if } x=y z, & y \in A_{\left(t_{3}, t_{3}^{\prime}\right)}-A_{\left(t_{2}, t_{2}^{\prime}\right)} \\
(0,1) & \text { otherwise } &
\end{array}\right.
$$

Therefore $D . C \subseteq A$, but $\left(\mu_{C}(a), v_{C}(a)\right)=\left(s_{1}, s_{1}^{\prime}\right)>\left(t_{1}, t_{1}^{\prime}\right)=$ $\left(\mu_{A}(a), v_{A}(a)\right)$ and $\left(\mu_{D}(a), v_{D}(a)\right)=\left(s_{2}, s_{2}^{\prime}\right)>\left(t_{2}, t-2^{\prime}\right)=$ $\left(\mu_{A}(b), v_{A}(b)\right)$. Thus $C \neq A, D \neq A$ a contradiction.

If $\operatorname{ImA}=\left\{\left(t_{1}, t_{1}^{\prime}\right),\left(t_{2}, t_{2}^{\prime}\right)\right\}$ for $(1,0)>\left(t_{1}, t_{1}^{\prime}\right)>\left(t_{2}, t_{2}^{\prime}\right) \geq(0,1)$, then there exist $d, e \in N$ such that

$\left(\mu_{A}(d), v_{A}(d)\right)=\left(t_{1}, t_{1}^{\prime}\right),\left(\mu_{A}(e), v_{A}(e)\right)=\left(t_{2}, t_{2}^{\prime}\right)$. Choose $s_{1}, s_{2}$ in such a way that $(1,0)>\left(s_{1}, s_{1}^{\prime}\right)>\left(t_{1}, t_{1}^{\prime}\right)>\left(s_{2}, s_{2}^{\prime}\right)>\left(t_{2}, t_{2}^{\prime}\right)$. Define $C(x)=\left(\mu_{C}(x), v_{C}(x)\right)$ and $D(x)=\left(\mu_{D}(x), v_{D}(x)\right)$ as

$$
C(x)= \begin{cases}\left(s_{1}, s_{1}^{\prime}\right) & \text { if } x \in A_{\left(t_{1}, t_{1}^{\prime}\right)} \\ \left(t_{2}, t_{2}^{\prime}\right) & \text { otherwise }\end{cases}
$$




$$
D(x)= \begin{cases}\left(t_{1}, t_{1}^{\prime}\right) & \text { if } x \in A_{\left(t_{1}, t_{1}^{\prime}\right)} \\ \left(s_{2}, s_{2}^{\prime}\right) & \text { otherwise }\end{cases}
$$

Clearly $A \subseteq C, A \subseteq D$.

$(D . C)(x)= \begin{cases}\left(t_{1}, t_{1}^{\prime}\right) \quad \text { if } x=y z & y, z \in A_{\left(t_{1}, t_{1}^{\prime}\right)} \\ \left(s_{2}, s_{2}^{\prime}\right) \quad \text { if } x=y z, & y \in A_{\left(t_{2}, t_{2}^{\prime}\right)}-A_{\left(t_{1}, t_{1}^{\prime}\right)}, \\ z \in A_{\left(t_{1}, t_{1}^{\prime}\right)} & \\ \left(t_{2}, t_{2}^{\prime}\right) \quad \text { if } x=y z, & y, z \notin A_{\left(t_{1}, t_{1}^{\prime}\right)} \\ (0,1) \quad \text { otherwise } & \end{cases}$

Thus $D . C \subseteq A$, but $C(d)=\left(s_{1}, s_{1}^{\prime}\right)>\left(t_{1}, t_{1}^{\prime}\right)=A(a), \quad D(e)=$ $\left(s_{2}, s_{2}^{\prime}\right)>\left(t_{2}, t_{2}^{\prime}\right)=A(b)$. Therefore $C \neq A \quad D \neq A$ a contradiction. Therefore $\operatorname{Im} A=\left\{(1,0),\left(t, t^{\prime}\right)\right\}$ with $t+t^{\prime} \leq 1$, where $(1,0)>\left(t, t^{\prime}\right) \geq(0,1)$.

Lemma 3.14. If $A$ is a non constant IF weakly prime ideals of $N$, then $A_{(1,0)}$ is a prime left ideal on $N$.

Proof. Let $A$ be a non constant IF weakly prime ideal of $N$. Let $C, D$ be left ideals of $N$ such that $C . D \subseteq A_{(1,0)}, A_{(1,0)} \subseteq C$ and $A_{(1,0)} \subseteq D$. Define IFSs $I, J$ as

$I(x)=\left\{\begin{array}{ll}(1,0) & \text { if } x \in C \\ \left(t, t^{\prime}\right) & \text { otherwise }\end{array} \quad J(x)= \begin{cases}(1,0) & \text { if } x \in D \\ \left(t, t^{\prime}\right) & \text { otherwise }\end{cases}\right.$

Clearly $I, J$ are IF strong left ideals of $N, A \subseteq I, \quad A \subseteq J$.

$$
(I . J)(x)= \begin{cases}(1,0) & \text { if } x \in C . D \\ \left(t, t^{\prime}\right) & \text { if } x=y z, y \notin \operatorname{Cor} z \notin D \\ (0,1) & \text { otherwise }\end{cases}
$$

Therefore $I . J \subseteq A$ implies $I=A$ or $J=A$. Thus $C=I$ or $D=J$. Hence $A_{(1,0)}$ is a weakly prime left ideal of $N$.

Theorem 3.15. Let $A$ be a non constant IF left ideal of $N$. Then $A$ is an IF weakly prime left ideal of $N$ if and only if

(i) $\operatorname{Im} A=\left\{(1,0),\left(t, t^{\prime}\right)\right\}$, where $(1,0)>\left(t, t^{\prime}\right) \geq(0,1)$.

(ii) $A_{(1,0)}$ is a weakly prime left ideal of $N$.

Proof. If $A$ is an IF weakly prime left ideal of $N$, then $(i),(i i)$ is true from Lemma 3.13 and Lemma 3.14.

Conversely, if there exist IF left ideals $I, J$ of $N$ containing $A$ such that $I . J \subseteq A$ with $I \neq A$ and $J \neq A$. Therefore there exist $a, b \in N$ such that $I(a)=\left(s_{1}, s_{1}^{\prime}\right)>\left(t, t^{\prime}\right)=A(a)$ and $J(b)=\left(s_{2}, s_{2}^{\prime}\right)>\left(t, t^{\prime}\right)=A(b)$. Thus $a \in I_{\left(s_{1}, s_{1}^{\prime}\right)}$ but $a \notin$ $A_{(1,0)}$ and $b \in J_{\left(s_{2}, s_{2}^{\prime}\right)}$ but $b \notin A_{(1,0)}$. Clearly $I_{\left(s_{1}, s_{1}^{\prime}\right)}, J_{\left(s_{2}, s_{2}^{\prime}\right)}$ are strong left ideals. Let $x \in A_{(1,0)}$. As $A \subseteq I, A(x)=(1,0)$, then $I(x)=(1,0)$. Thus $I(x) \geq\left(s_{1}, s_{1}^{\prime}\right)$. Therefore $A_{(1,0)} \subseteq I_{\left(s_{1}, s_{1}^{\prime}\right)}$. Similarly, $A_{(1,0)} \subseteq J_{\left(s_{2}, s_{2}^{\prime}\right)}$. If $I_{\left(s_{1}, s_{1}^{\prime}\right)} \cdot J_{\left(s_{2}, s_{2}^{\prime}\right)} \subseteq$, then $I_{\left(s_{1}, s_{1}^{\prime}\right)}=$ $A_{(1,0)}$ or $A_{(1,0)}=J_{\left(s_{2}, s_{2}^{\prime}\right)}$ which is a contradiction. Thus $I_{\left(s_{1}, s_{1}^{\prime}\right)} \cdot J_{\left(s_{2}, s_{2}^{\prime}\right)} \not A_{(1,0)}$. Then $c d \notin A_{(1,0)}$ for some $c \in I_{\left(s_{1}, s_{1}^{\prime}\right)}$ and for some $d \in J_{\left(s_{2}, s_{2}^{\prime}\right)}$. Thus $A(c d)=\left(t, t^{\prime}\right)$.

$(I . J)(c d) \geq I(c) \wedge J(d)=\left(s_{1}, s_{1}^{\prime}\right) \wedge\left(s_{2}, s_{2}^{\prime}\right)>\left(t, t^{\prime}\right)=A(c d)$. Which is a contradiction to $I . J \subseteq A$. Therefore $A$ is an IF weakly prime left ideals of $N$.

Theorem 3.16. Let A be a non-constant IF strong left ideal of $N$. Then $A$ is an IF prime left ideal of $N$ if and only if

(i) $I M A=\left\{(1,0),\left(t, t^{\prime}\right)\right\}$

(ii) $A_{(1,0)}$ is a prime left ideal of $N$.

Proof. Let $A$ be an IF prime left ideal of $N$. Then $A$ is an IF weakly prime left ideal of $N$. From Lemma 3.13 IMA $==$ $\left\{(1,0),\left(t, t^{\prime}\right)\right\}$, where $(1,0)>\left(t, t^{\prime}\right) \geq(0,1)$. Let $C, D$ be strong left ideals of $N$ such that $C . D \subseteq A_{(1,0)}$. Then $\chi_{C}, \chi_{D}$ are IF strong left ideals of $N$.

$$
\left(\chi_{C} \cdot \chi_{D}\right)(x)= \begin{cases}(1,0) & \text { if } x \in C . D \\ (0,1) & \text { otherwise }\end{cases}
$$

Therefore $\chi_{C} \cdot \chi_{D} \subseteq A$ and so $\chi_{C} \subseteq A$ or $\chi_{D} \subseteq A$. Hence $C \subseteq A$ or $D \subseteq A$. Hence $A_{(1,0)}$ is a prime left ideals of $N$.

Conversely, if there exist IF left ideals $I, J$ of $N$ containing $A$ such that $I . J \subseteq A$ with $I \nsubseteq A$ and $J \nsubseteq A$. Therefore there exist $a, b \in N$ such that $I(a)=\left(s_{1}, s_{1}^{\prime}\right)>\left(t, t^{\prime}\right)=A(a)$ and $J(b)=\left(s_{2}, s_{2}^{\prime}\right)>\left(t, t^{\prime}\right)=A(b)$. Thus $a \in I_{\left(s_{1}, s_{1}^{\prime}\right)}$ but $a \notin$ $A_{(1,0)}$ and $b \in J_{\left(s_{2}, s_{2}^{\prime}\right)}$ but $b \notin A_{(1,0)}$. Clearly $I_{\left(s_{1}, s_{1}^{\prime}\right)}, J_{\left(s_{2}, s_{2}^{\prime}\right)}$ are strong left ideals. Let $x \in A_{(1,0)}$. As $A \subseteq I, A(x)=(1,0)$, then $I(x)=(1,0)$. Thus $I(x) \geq\left(s_{1}, s_{1}^{\prime}\right)$. Therefore $A_{(1,0)} \subseteq I_{\left(s_{1}, s_{1}^{\prime}\right)}$. Similarly, $A_{(1,0)} \subseteq J_{\left(s_{2}, s_{2}^{\prime}\right)}$. If $I_{\left(s_{1}, s_{1}^{\prime}\right)} \cdot J_{\left(s_{2}, s_{2}^{\prime}\right)} \subseteq$, then $I_{\left(s_{1}, s_{1}^{\prime}\right)} \subseteq$ $A_{(1,0)}$ or $A_{(1,0)} \subseteq J_{\left(s_{2}, s_{2}^{\prime}\right)}$ which is a contradiction. Thus $I_{\left(s_{1}, s_{1}^{\prime}\right)} \cdot J_{\left(s_{2}, s_{2}^{\prime}\right)} \not \subset A_{(1,0)}$. Then $c d \notin A_{(1,0)}$ for some $c \in I_{\left(s_{1}, s_{1}^{\prime}\right)}$ and for some $d \in J_{\left(s_{2}, s_{2}^{\prime}\right)}$. Thus $A(c d)=\left(t, t^{\prime}\right)$.

$(I . J)(c d) \geq I(c) \wedge J(d)=\left(s_{1}, s_{1}^{\prime}\right) \wedge\left(s_{2}, s_{2}^{\prime}\right)>\left(t, t^{\prime}\right)=A(c d)$. Which is a contradiction to $I . J \subseteq A$. Therefore $A$ is an IF prime left ideals of $N$.

\section{Conclusion}

In this paper the notion of Intuitionistic fuzzy ideals of a near-subtraction semigroup are presented and properties of these ideals are discussed.

\section{References}

[1] S. Abou-Zaid, On fuzzy subnear-ring and ideals, Fuzzy sets and Systems, 44 (1991), 139-146.

[2] K.T. Atanasov, Intuitionstic fuzzy sets, J. Fuzzy Math. 20 (1) (1986), 87-96.

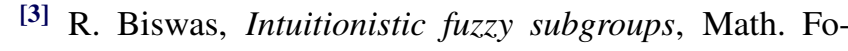
rum.10 (1987), 37-46.

[4] Y.U. Cho and Y.B. Jun, On intuitionistic fuzzy $R$ subgroups of near-rings, J. Appl. Math. Comput. 18 (2005), 665-677. 
[5] P.Dheena and G.Mohanraj,On prime and fuzzy prime fuzzy ideals of subtraction algebra, Int.Math.Forum 4(47)(2009), 2345-2353.

[6] P.Dheena and G.Satheeshkumar,On strongly regular near-subtraction semi-groups, Commun.Korean Math.Soc.22(3)(2007) 323-330.

[7] Y.B. Jun, K.H. Kim and Y.H. Yon. Intuitionistic fuzzy ideals of near-rings, J. Inst. Math. Comput. Sci., Math. Ser. 12 (1999), 221-228.

[8] Y.B.Jun,H.S.Kim and E.H.Roh,Ideal theory of subtraction algebras, Sci.Math.Jpn.61(3)(2005)459-464.

[9] Liu, W. Fuzzy invariant subgroups and Fuzzy ideals, Fuzzy Sets and System, 8, 133-139.

[10] O. Kazanci, S. Yamak and S. Yilmaz. On intuitionistic $Q$ fuzzy $R$-subgroups of near-rings, Int. Math. Forum, 2(59) (2007), 2899-2910.

[11] K.H. Kim, On intuitionistic Q-fuzzy semiprime ideals in semigroups, Adv. Fuzzy Math.1 (2006), 15-21.

[12] Manikantan, T. fuzzy bi-ideals of near-rings, Journal of Fuzzy Mathematics, 17 ,(3)(2009), 659-671.

[13] A. Rosenfeld, Fuzzy groups, Journal of Mathematical Analysis and Application, 35 (1971), 512-517.

[14] Saikia H.K. and L.K Barthakur, On fuzzy N-subgroups and fuzzy ideals of near-rings and near-ring groups, $\mathrm{J}$. Fuzzy Mathematics, 11,(3)(2003), 567-580.

[15] B.M.Schein,Difference semigroups, Comm.Algebra,20(1992),2153-2169.

[16] T. Tamizh Chelvam and N. Ganesan, On bi-ideals of nearrings, Indian J.pure appl.Math., 18,(11)(1987), 10021005.

[17] L.A. Zadeh, Fuzzy Sets, Information and Control, 8 (1965) 338-353.

[18] B.Zelinka,Subtraction semigroups, Math.Bohemica,120(1995), 445-447.

$\operatorname{SSN}(\mathrm{P}): 2319-3786$

Malaya Journal of Matematik

$\operatorname{ISSN}(\mathrm{O}): 2321-5666$

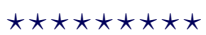

